\title{
MAGNETIC FIELD LINE RANDOM WALK IN MODELS AND SIMULATIONS OF REDUCED MAGNETOHYDRODYNAMIC TURBULENCE
}

\author{
A. P. Snodin ${ }^{1,2,3}$, D. Ruffolo ${ }^{1,2}$, S. Oughton ${ }^{4}$, S. Servidio ${ }^{5}$, and W. H. Matthaeus ${ }^{6}$ \\ ${ }^{1}$ Department of Physics, Faculty of Science, Mahidol University, Bangkok 10400, Thailand; andrew.snodin@gmail.com \\ 2 Thailand Center of Excellence in Physics, CHE, Ministry of Education, Bangkok 10400, Thailand \\ ${ }^{3}$ Department of Materials and Production Technology Engineering, Faculty of Engineering, King Mongkut's \\ University of Technology North Bangkok, Bangkok 10800, Thailand \\ ${ }^{4}$ Department of Mathematics, University of Waikato, Hamilton 3240, New Zealand \\ ${ }^{5}$ Dipartimento di Fisica, Università della Calabria, I-87036 Cosenza, Italy \\ ${ }^{6}$ Bartol Research Institute and Department of Physics and Astronomy, University of Delaware, Newark, DE 19716, USA \\ Received 2013 May 21; accepted 2013 October 11; published 2013 November 25
}

\begin{abstract}
The random walk of magnetic field lines is examined numerically and analytically in the context of reduced magnetohydrodynamic (RMHD) turbulence, which provides a useful description of plasmas dominated by a strong mean field, such as in the solar corona. A recently developed non-perturbative theory of magnetic field line diffusion is compared with the diffusion coefficients obtained by accurate numerical tracing of magnetic field lines for both synthetic models and direct numerical simulations of RMHD. Statistical analysis of an ensemble of trajectories confirms the applicability of the theory, which very closely matches the numerical field line diffusion coefficient as a function of distance $z$ along the mean magnetic field for a wide range of the Kubo number $R$. This theory employs Corrsin's independence hypothesis, sometimes thought to be valid only at low $R$. However, the results demonstrate that it works well up to $R=10$, both for a synthetic RMHD model and an RMHD simulation. The numerical results from the RMHD simulation are compared with and without phase randomization, demonstrating a clear effect of coherent structures on the field line random walk for a very low Kubo number.
\end{abstract}

Key words: diffusion - magnetic fields - magnetohydrodynamics (MHD) - turbulence

\section{INTRODUCTION}

The random wandering of magnetic field lines in a turbulent medium can be studied by using models or simulations of the magnetic fluctuations and then computing the integral curves (field lines) at a fixed instant of time, as illustrated in Figure 1. For fluctuations transverse to a mean field direction $z$, perpendicular displacements such as $\Delta x(z)$ are treated as random variables. Under a wide range of suitable conditions, the ensemble average of mean square displacements reaches a diffusive limit, $\left\langle[\Delta x(z)]^{2}\right\rangle \sim 2 D z$ with a "diffusion coefficient" $D$. This field line random walk (FLRW) was originally treated in the quasi-linear limit (Jokipii 1966) and has significant implications for the galactic confinement of cosmic rays (Jokipii \& Parker 1969b), particle acceleration at perpendicular shocks (Giacalone \& Jokipii 1996), solar energetic particle transport (Giacalone et al. 2000; Ruffolo et al. 2003; Zimbardo et al. 2004), heat conduction by electrons (Chandran \& Cowley 1998; Lazarian 2006), and many other astrophysical applications. FLRW has notable implications for particle diffusion, especially perpendicular diffusion (Rechester \& Rosenbluth 1978; Giacalone \& Jokipii 1999; Matthaeus et al. 2003), as well as the problem of magnetic reconnection in the solar corona (Lazarian \& Vishniac 1999; Rappazzo et al. 2012). Extensions of the theory beyond the quasi-linear approximation include the implementation of nonperturbative methods (Taylor \& McNamara 1971; Matthaeus et al. 1995), typically based on Corrsin's hypothesis (Corrsin 1959) (see the Appendix), which form the basis for much of the approach adopted herein. Here we test FLRW theory for weakly three-dimensional (3D; i.e., quasi-two-dimensional) models of fluctuating magnetic fields in the context of reduced magnetohydrodynamics (RMHD; Strauss 1976; Kadomtsev \& Pogutse
1979; Montgomery 1982). The RMHD model of magnetic turbulence is appropriate at low plasma beta (the ratio of kinetic to magnetic pressure), under the assumption that the motions remain nearly incompressible (Zank \& Matthaeus 1992). It is most appropriate, for example, in systems such as coronal flux tubes, where it is routinely used for studies of coronal heating in both closed (Einaudi et al. 1996; Dmitruk \& Gómez 1997) and open field line regions (Dmitruk \& Matthaeus 2003).

In the present work, we discuss and test the validity of a recently developed theory of FLRW in RMHD (Ruffolo \& Matthaeus 2013) and carry out a statistical study of this phenomenon using synthetic model fields as well as RMHD solutions. We examine ensembles of field lines obtained numerically, and evaluate the accuracy of the theory for a range of Kubo numbers $R=\left(b / B_{0}\right)\left(\ell_{c} / \ell_{\perp}\right)$, where $b$ is the rms magnetic fluctuation strength, $B_{0}$ is the mean magnetic field, and $\ell_{c}$ and $\ell_{\perp}$ are the correlation lengths in the parallel and perpendicular directions, respectively. We find that three slightly different variations of the theory agree well with the numerical determinations of the diffusion coefficient. This theory is based on the Corrsin hypothesis, sometimes presumed to be valid only at low Kubo numbers (e.g., Vlad et al. 1998; Neuer \& Spatschek 2006; Negrea et al. 2007). Somewhat surprisingly, we find that the theory remains accurate from the low Kubo number (quasi-linear) regime up to Kubo numbers well in excess of unity.

The structure of the paper is as follows. In Section 2, we first focus (Section 2.1) on the RMHD model, briefly summarizing its kinematic and dynamical properties; then, (Section 2.2) we briefly review FLRW theories and the recently published theory of FLRW in RMHD (Ruffolo \& Matthaeus 2013). Section 3 describes the methods we employ-a synthetic model of RMHD fields (Section 3.1), RMHD simulations (Section 3.2), and 


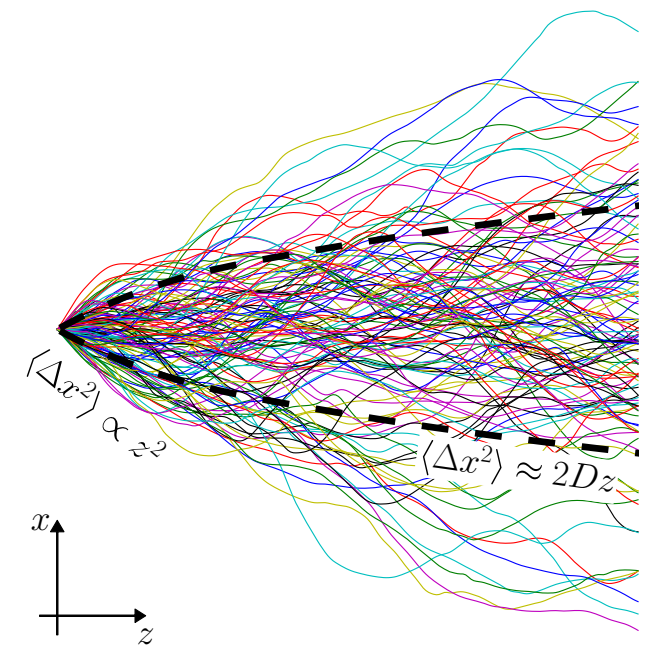

Figure 1. Collection of turbulent magnetic field lines (solid lines) generated using a synthetic RMHD model, shown as originating at the same point, along with the envelope at $\pm \Delta x_{\text {rms }}$ (dashed lines) that encompasses $\approx 68 \%$ of field lines. At low $z$ there is a free-streaming regime where field lines are nearly straight lines so that $\left\langle\Delta x^{2}\right\rangle \propto z^{2}$ and $\Delta x_{\mathrm{rms}} \propto z$. Then there is a transition to a high- $z$ diffusive regime where $\left\langle\Delta x^{2}\right\rangle \approx 2 D z$ and $\Delta x_{\text {rms }} \propto \sqrt{z}$. In the present work we numerically evaluate the field line diffusion coefficient $D$ and compare this with theoretical expectations.

field line tracing (Section 3.3). Section 4 provides the main results of the study-Section 4.1 describes the results for a synthetic RMHD field, which is essentially a random-phase initial condition for RMHD with a specified spectrum, while Section 4.2 presents results for a dynamically evolved solution of the RMHD equations. In each case, three versions of the FLRW theory are compared with statistics computed from an ensemble of field lines obtained from the model or simulation fields. Finally, the main conclusions are discussed in Section 5, along with implications for future work.

\section{THEORETICAL BACKGROUND}

\subsection{Reduced Magnetohydrodynamic Turbulence}

For low plasma beta, an externally supported mean magnetic field $\mathbf{B}_{0}$ that is strong compared to rms fluctuations in the magnetic field $b$ and velocity field $u$ and nearly incompressible conditions, one can obtain a reduced form of magnetohydrodynamics (RMHD) with the following properties. First, with $b \sim u$ (in Alfvén units) and defining $\delta b / B_{0}=O(\epsilon)$ (small), all dynamically varying quantities are functions of all three Cartesian coordinates $x, y, z$; however, the fluctuations are slowly varying in $z$ in the sense that $\partial / \partial x \sim \partial / \partial y=O(1)$ while $\partial / \partial z=O(\epsilon)$. Then the magnetic field can be decomposed as

$$
\boldsymbol{B}=B_{0} \hat{z}+\nabla a \times \hat{z}=\boldsymbol{B}_{0}+\boldsymbol{b},
$$

where $\hat{z}$ is the direction of the uniform mean magnetic field, $\boldsymbol{b}$ is a transverse fluctuation, and $a=a(x, y, \epsilon z)$ is a magnetic potential that varies slowly in the $z$-direction. Both magnetic and velocity fluctuations are slowly varying in $z$ and transverse to the mean field $\left(u_{z}=b_{z}=0\right)$. The latter condition is particularly important for obtaining RMHD behavior from full MHD (Dmitruk et al. 2005). Other theoretical representations of MHD turbulence (e.g., Goldreich \& Sridhar 1995) adopt a representation having fluctuations transverse to a mean field, which, in MHD wave theory, is known as the "Alfvén mode" (see also Dobrowolny et al. 1980; Zank \& Matthaeus 1992;
Lithwick \& Goldreich 2001; Cho \& Lazarian 2002; Cho et al. 2002; Kowal \& Lazarian 2010).

In terms of Alfvén speed units, and introducing the perpendicular gradient operator $\nabla_{\perp}=\left(\partial_{x}, \partial_{y}, 0\right)$, we may then write the RMHD equations as

$$
\begin{aligned}
& \partial_{t} \mathbf{u}+\mathbf{u} \cdot \nabla_{\perp} \mathbf{u}=-\nabla_{\perp} P+\mathbf{b} \cdot \nabla_{\perp} \mathbf{b}+B_{0} \partial_{z} \mathbf{b}+v \nabla_{\perp}^{2} \mathbf{u}, \\
& \partial_{t} \mathbf{b}+\mathbf{u} \cdot \nabla_{\perp} \mathbf{b}=\mathbf{b} \cdot \nabla_{\perp} \mathbf{u}+B_{0} \partial_{z} \mathbf{u}+\mu \nabla_{\perp}^{2} \mathbf{b},
\end{aligned}
$$

with $\nabla_{\perp} \cdot \mathbf{u}=\nabla_{\perp} \cdot \mathbf{b}=0$, and for resistivity $\mu$ and viscosity $v$. In the following sections, we employ (1) synthetic fields that have properties of RMHD fields as described above, which are not evolved using Equations (2) and (3), but would be suitable as initial data; and (2) an RMHD solution, which is obtained as the output of a simulation code that solves the above equations. Both of these are described further below.

\subsection{Field Line Random Walk}

For our synthetic and simulated RMHD fields, the fluctuations are designed to be realizations of random fields that are statistically homogeneous in space and time and are axisymmetric, i.e., the statistics of $x$ - and $y$-displacements are identical. The latter is a simplification for this initial exploration of the FLRW; there are physical situations where non-axisymmetry may be important, and non-axisymmetric Corrsin-based theories and simulations of field line and energetic particle diffusion have been developed for other field models (e.g., Ruffolo et al. 2006, 2008; Weinhorst et al. 2008). Because RMHD fluctuations are transverse, a field line never backtracks along $z$, and the field line trajectory can be specified by single-valued functions $x(z)$ and $y(z)$. These can be determined from

$$
\frac{d x}{d z}=\frac{b_{x}(x, y, z)}{B_{0}}, \quad \frac{d y}{d z}=\frac{b_{y}(x, y, z)}{B_{0}} .
$$

The problem of FLRW is to characterize the statistics of the displacement $\Delta x$ (or $\Delta y$ ) after a distance $z$ parallel to the mean magnetic field $\mathbf{B}_{0}$ from an ensemble of field line trajectories. Because the mean fluctuation is zero, the mean displacement is also zero. Thus, the most useful statistics are related to the variance $\left\langle\Delta x^{2}\right\rangle=\left\langle\Delta y^{2}\right\rangle$.

An illustration of FLRW behavior is shown in Figure 1. At low $z$, we typically expect field lines to have straight line (ballistic) trajectories with $\Delta x_{\mathrm{rms}}=\left(b_{x, \mathrm{rms}} / B_{0}\right) z$. Then, for a broad class of homogeneous systems with finite parallel correlation scale $\ell_{c}$, we expect that, when $z \gg \ell_{c}$, the increments of the displacement become uncorrelated, giving rise to a diffusive random walk with $\left\langle\Delta x^{2}\right\rangle=2 D_{\infty} \Delta z$, where $D_{\infty}$ is the asymptotic diffusion coefficient. Many studies have examined the scaling of this quantity and/or quantitative estimates of its value. It is also useful to examine a running diffusion coefficient $D(z)$ defined by

$$
D(z) \equiv \frac{1}{2} \frac{d}{d z}\left\langle\Delta x^{2}\right\rangle
$$

for which $D(z \rightarrow \infty)=D_{\infty}$. For example, a comparison with simulation results can be used to discriminate between different possible theoretical expressions, in which case a comparison of the function $D(z)$ is more powerful than comparing $D_{\infty}$ alone (Snodin et al. 2013).

The quantitative expression of Jokipii \& Parker (1969a) is known to apply for quasi-1D turbulence, i.e., a low Kubo 
number, $R \ll 1$, and can be expressed as

$$
D_{\infty}=\frac{1}{2} \frac{b^{2}}{B_{0}^{2}} \ell_{c}, \quad(R \ll 1),
$$

where $b^{2}$ is shorthand for $\left\langle b^{2}\right\rangle$, which represents the magnetic fluctuation energy, and $\ell_{c}$ is the correlation length along $z$. The scaling $D_{\infty} \propto\left(b / B_{0}\right)^{2}$ is referred to as quasi-linear scaling. For a high Kubo number, $R \gg 1$, the so-called Bohm scaling, $D_{\infty} \propto\left(b / B_{0}\right)$, was proposed by Kadomtsev $\&$ Pogutse (1979) and a percolative scaling $D_{\infty} \propto R^{0.7}$ was proposed by Isichenko (1991). Theories based on Corrsin's hypothesis (e.g., Matthaeus et al. 1995; Ruffolo et al. 2004) have quasi-linear scaling for fluctuation models dominated by parallel wave vectors (quasi-1D) and Bohm scaling for those dominated by perpendicular wave vectors (quasi-2D), whereas the decorrelation trajectory method of Vlad et al. (1998) can provide a scaling that differs from both Bohm and percolative scaling in the quasi-2D regime. Various numerical studies of the FLRW or analogous problems using singlecomponent models of turbulence described a transition from quasi-linear to percolative behavior (Ottaviani 1992; Zimbardo \& Veltri 1995; Reuss \& Misguich 1996; Zimbardo et al. 2000). Computer simulations by Hauff et al. (2010) for stretched isotropic turbulence examined the scaling exponent $\gamma$ so that $D_{\infty} \propto R^{\gamma}$ and found a variation from $\gamma \sim 2$ (quasi-linear scaling) for $R \lesssim 0.1$ down to $\gamma \sim 0.7$ (percolative scaling) for $R \gtrsim 10$. For $2 \mathrm{D}+$ slab fluctuations, simulations by Ghilea et al. (2011) indicated that Corrsin-based theories, involving a combination of quasi-linear and Bohm diffusion, worked quantitatively well in all cases examined, and modest deviations attributed to trapping or percolation effects were found in the limit of almost completely two-dimensional (2D) fluctuations.

For RMHD fluctuations as considered in this work, an analytic theory was developed by Ruffolo \& Matthaeus (2013), which, for the reader's convenience, is summarized in the Appendix. Based on the assumptions of Corrsin's hypothesis and a Gaussian displacement distribution, there are three versions of the theory based on diffusive decorrelation (DD), random ballistic decorrelation (RBD), and a second-order ordinary differential equation (ODE). The latter is sometimes implied in other works where simply "Corrsin approximation" is mentioned (e.g., Vlad et al. 1998). These three versions, described by Equations (A4) to (A7), exhibit quasi-linear scaling at $R \lesssim 1$ and Bohm diffusion at $R \gtrsim 1$. (A DD theory by Shalchi \& Kolly (2013) had similar scaling for an analytic field model designed to represent the numerical field model of Cho et al. (2002) and the critical balance concept of Goldreich \& Sridhar 1995.) These correspond to three possible closures of a general differential equation. Of these, the RBD version is simplest to use, involving a single explicit formula. For RBD, the variance of the displacement distribution is calculated assuming random ballistic trajectories (with variance proportional to $z^{2}$ ), which is most appropriate for low $z$ (Figure 1). The DD version involves solving an implicit equation for $D_{\infty}$, and the displacement distribution is found from the assumption of diffusion at the asymptotic rate, which is most appropriate for high $z$. The ODE version requires solving the differential equation as a function of $z$. This uses a self-consistent equation for the variance of the displacement distribution, which would seem to be appropriate for all $z$, but any approximation errors at lower $z$ become compounded with increasing $z$. These three versions of the theory will be tested in the present work.

\section{COMPUTATIONAL TECHNIQUES}

\subsection{Synthetic RMHD Field}

The synthetic RMHD magnetic field is constructed using random phases and a specified spectral amplitude so that it has the key properties of RMHD fields. This computationally straightforward approach is often used to generate initial conditions for RMHD simulations. Moreover, it yields explicit forms for the spectra, facilitating direct comparison with theories.

Following Ruffolo et al. (2004) and Ruffolo \& Matthaeus (2013), we use

$$
\tilde{a}\left(\mathbf{k}_{\perp}, k_{z}\right) \propto \begin{cases}{\left[1+\left(\frac{k_{\perp}}{k_{0}}\right)^{2}\right]^{-7 / 6} e^{i \varphi(\mathbf{k})}} & \left|k_{z}\right| \leqslant K \\ 0 & \text { otherwise }\end{cases}
$$

where $\tilde{a}$ is the Fourier transform of the magnetic potential, $\mathbf{k}_{\perp} \equiv\left(k_{x}, k_{y}\right)$ is the wave vector perpendicular to the mean magnetic field, $k_{z}$ is the parallel wave vector, and $\varphi(\mathbf{k})$ is a phase that is independently random for each Fourier mode k. This potential function is substituted into Equation (1) to yield the magnetic field, which is then normalized to produce the desired magnetic energy $b^{2}$. Note that the above functional form leads to a magnetic power (energy) spectrum in the inertial range that is consistent with the Kolmogorov law $k_{\perp}^{-5 / 3}$, and has a boxcar profile along $k_{z}$. We choose the gauge in which $\tilde{a}\left(\mathbf{k}_{\perp}=0, k_{z}\right)=$ 0 , and the magnetic potential satisfies the Hermitian reality condition $\tilde{a}\left(\mathbf{k}_{\perp}, k_{z}\right)=\tilde{a}^{*}\left(-\mathbf{k}_{\perp},-k_{z}\right)$. With random phases, the magnetic field components have distributions very close to Gaussian, lacking the coherent structures and intermittency that are characteristic of the cascade in RMHD turbulence (Einaudi et al. 1996; Veltri 1999).

We define the size of the periodic spatial domain to be $2 \pi L_{z}$ in the $z$-direction and $2 \pi L_{\perp}$ in the $x$ - and $y$-directions, so that $L_{\perp}=L_{x}=L_{y}$. Then the Fourier modes $k_{i}$ along the $i$-direction are integral multiples of $1 / L_{i}$. We use a $(1024)^{3}$ mesh and set $k_{0} L_{\perp}=6$, which establishes the bendover scale of the spectrum in the perpendicular direction, while $K L_{z}=25$ represents the parallel cutoff. These characteristic wavenumbers control the perpendicular correlation length $\ell_{\perp}$, which we identify with the total correlation length $\lambda_{c 2}$ as defined by Matthaeus et al. (2007), and the parallel correlation length $\ell_{c} \equiv \int_{0}^{\infty} R_{x x}^{r}(z) d z / R_{x x}^{r}(0)$, where $R_{x x}^{r}(z)=R_{y y}^{r}(z)$ is the reduced correlation function along $z$. With the above choices, a good compromise is reached in resolving the correlation length, representing an inertial range, and achieving statistical homogeneity of the turbulence, with over 10 mesh points per correlation length. In terms of correlation scales, the periodic box is large, with $\sim 100$ correlation lengths in both the parallel and perpendicular directions.

\subsection{RMHD Simulation}

We compute solutions to the RMHD Equations (2) and (3) by employing a pseudospectral method and a vorticity-magnetic potential representation (see, e.g., Oughton et al. 2004). The initial condition is a synthetic field, as described in the previous subsection, using random phases by choosing Fourier amplitudes with Gaussian distributions for the real and imaginary parts that on average yield the specified squared amplitude, and keeping only modes with $4 \leqslant k_{\perp} L_{\perp} \leqslant 150$. The upper cutoff is comfortably less than the Galerkin limit, and the lower cutoff leaves some space for structures to grow according to the 


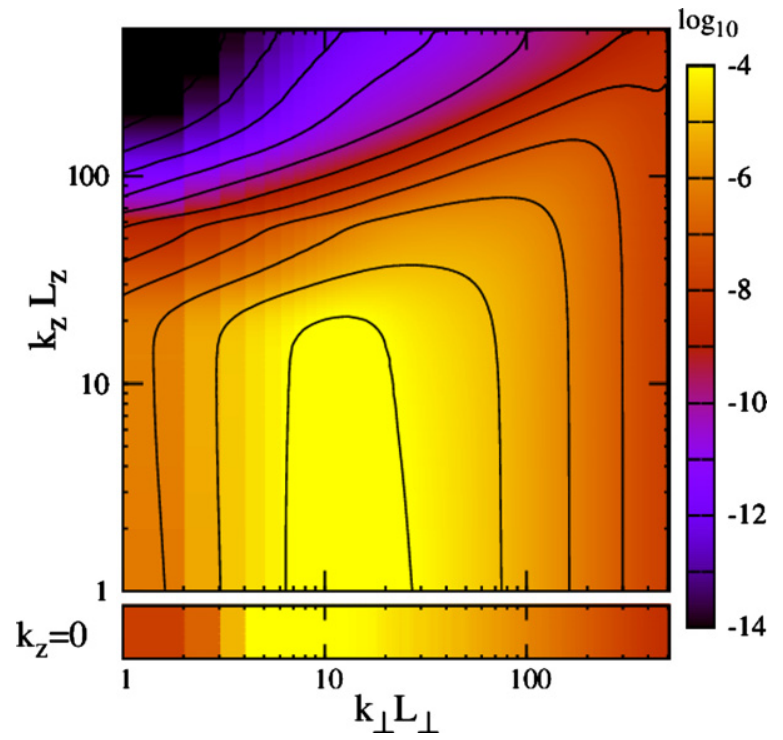

Figure 2. Surface plot of simulated RMHD magnetic spectrum as a function of $k_{\perp}$ and $k_{z}$. Spectral amplitude is according to the color scale shown in the legend, with several representative isospectral contours included for clarity. See text for details.

dynamics. The dynamical simulation includes no forcing, so it models the physics of turbulence evolution and decay. The time advancement, via a second-order accurate Runge-Kutta scheme, is carried out until time $t=0.6$, where time is expressed in units of $L_{\perp} / u_{0}$ and $u_{0}$ is set to the root-mean-squared speed of the plasma. The time $\mathrm{t}=0.6$ roughly corresponds to a non-linear time for the energy-containing structures present in the initial conditions. Thus, the system has been evolving long enough to establish a turbulent cascade and associated correlations, and the statistical properties at inertial and dissipation range scales are similar to those in stationary (driven) turbulence with the same Reynolds numbers. For efficiency, in view of the slow spread of energy in $k_{\|}$, the spatial resolution begins at $1024 \times 1024 \times 128$ and at $t=0.5$ expands to the final resolution of $1024^{3}$.

Figure 2 shows the magnetic energy spectrum (indicated by the color scale) after the RMHD simulation reached $t=0.6$, as a function of $\left(k_{\perp}, k_{z}\right)$. The strip at the bottom represents the energy spectrum as a function of $k_{\perp}$ for $k_{z}=0$. Note that the initial spectrum at $t=0$ only had energy in the wavenumber band $k_{z} L_{z} \leqslant K L_{z}=25$ and $4 \leqslant k_{\perp} L_{\perp} \leqslant 150$, with a Kolmogorov spectrum for $k_{\perp} L_{\perp} \gg k_{0} L_{\perp}=6$. After the RMHD simulation to $t=0.6$, the turbulent cascade has distributed energy to higher $k_{\perp}$. With increasing $k_{\perp}$, the energy is distributed over a wider range of $k_{z}$ values, which is qualitatively consistent with models of critical balance (Goldreich \& Sridhar 1995).

\subsection{Field Line Tracing}

For a 3D realization of magnetic turbulence on a discrete spatial grid, we trace magnetic field lines using a computer program based on that described by Dalena et al. (2012) and obtain running diffusion coefficients from the magnetic field line statistics. For a fixed RMHD fluctuation field, we may choose $B_{0}=(b / R)\left(l_{c} / l_{\perp}\right)$ in order to obtain the desired Kubo number $R$, which is the only parameter that controls the FLRW in dimensionless units. The parameters $b, l_{c}$, and $l_{\perp}$ are calculated from the input magnetic field data. We obtain a sample of field line trajectories by solving Equation (4) for 1000 independently random starting locations within the $3 \mathrm{D}$ box. Between grid points, trilinear interpolation is used to determine $b_{x}$ and $b_{y}$. We integrate the field line equations numerically using the fifthorder method of Cash \& Karp (1990), which allows for an adaptive step size via the error estimate of the fourth-order solution.

We impose triply periodic boundary conditions for the fields, to be consistent with all the realizations considered. Even if $\sqrt{\left\langle\Delta x^{2}\right\rangle}$ were to extend over several periodic box lengths in the $x$ - and $y$-directions, we would not expect significant box periodicity effects on the FLRW, because the box contains $\sim 100$ perpendicular correlation lengths. Thus, even when a trajectory exits and re-enters the box, it will most likely experience distinct turbulent structures. Furthermore, the turbulence models employed are 3D, so that even if the FLRW returns to the same $(x, y)$ at later $z$, the fluctuating field will be different from that at earlier $z$. In practice, it turns out that $\sqrt{\left\langle\Delta x^{2}\right\rangle}$ is always significantly less than the perpendicular box length by the time the FLRW becomes diffusive. However, periodic effects in $z$ are of concern because there is a finite probability that a field line trajectory will remain near the same $(x, y)$, in which case the fluctuating field will be highly correlated after displacement over a parallel box length. Therefore, in the present work, we trace field lines over a $z$-distance no greater than half the parallel box length, which is sufficient to study asymptotic diffusion.

From $\left\langle\Delta x^{2}\right\rangle$ as a function of $z$, we can obtain the running diffusion coefficient $D(z)$ by using Equation (5). The $\Delta x$ and $\Delta y$ statistics should be identical due to axisymmetry, so we also include statistics from $\Delta y$ in calculating $D(z)$. Quantities shown from magnetic field line tracing calculations are dimensionless, with $x$ and $z$ in units of $\ell_{\perp}$ and $\ell_{c}$, respectively, for the corresponding magnetic field representation. Then $D$ is in units of $\ell_{\perp}^{2} / \ell_{c}$. Comparing with the primed (dimensionless) units of Ruffolo \& Matthaeus (2013), where $K$ (see Equation (7)) is expressed in terms of $\ell_{c}$, the relevant conversions to our units are $x=x^{\prime}, z=z^{\prime} /(\pi / 2)$, and $D=(\pi / 2) D^{\prime}$. Theoretical predictions are always evaluated for the actual power spectrum of the magnetic field under consideration, with integrals over $\mathbf{k}$ converted to sums over Fourier modes.

\section{NUMERICAL RESULTS FOR THE FIELD LINE RANDOM WALK}

\subsection{Synthetic RMHD Field}

We first consider the case of a synthetic RMHD field as described in Section 3.1. In Figure 3 we compare the $z$ dependence of the running diffusion coefficient from numerical field line tracing with that of the Corrsin-based theory of Ruffolo $\&$ Matthaeus (2013) for various values of the Kubo number $R$. For this type of field model, there are oscillations in $D$ as a function of $z$, and the ability of a theory to match such oscillations in comparison with numerical results provides a much more stringent test than comparing with the asymptotic diffusion coefficient alone. At $R=0.1$, all three versions of the theory (which correspond to three different closures of a differential equation; see the Appendix) can match the numerical results very well, including the oscillations of $D(z)$. The DD version provides the best match, within a few percent. At $R=1$, the RBD and ODE versions provide a very good match to $D(z)$, while the DD version does not match as well, but is still within $\sim 15 \%$ of the numerical result.

These results suggest that Corrsin's hypothesis is working very well at low $R$. The Kubo number $R=\left(b / B_{0}\right)\left(\ell_{c} / \ell_{\perp}\right)$ expresses the ratio between the correlation scale along $z$, which 

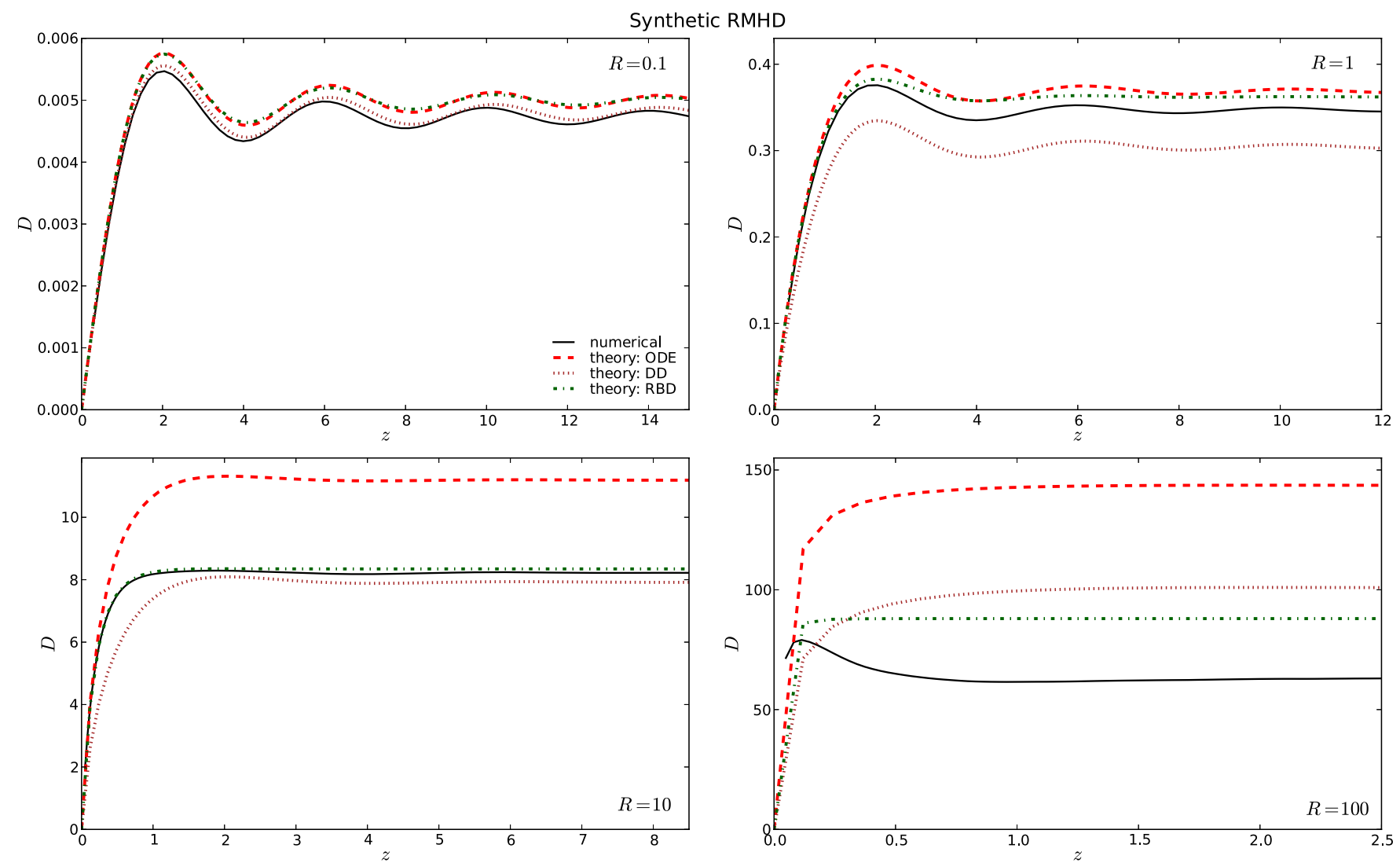

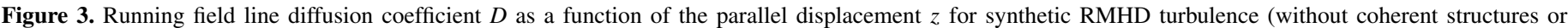

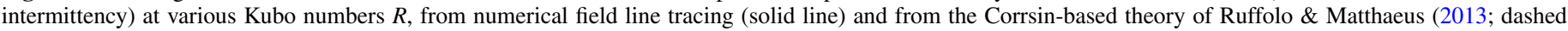

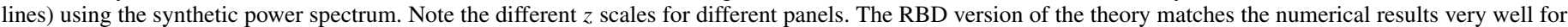

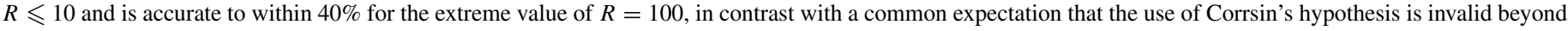
$R=1$.

characterizes an extrinsic decorrelation along $z$ that does not depend on the FLRW, and the correlation scale along $x$ or $y$, describing an intrinsic decorrelation that depends on the FLRW itself; the factor of $b / B_{0}$ serves to convert $\ell_{c}$ to a scale along $x$ or $y$ that can be directly compared with $\ell_{\perp}$. At low $R$, all versions of the theory work well because extrinsic decorrelation dominates and the FLRW itself has little effect. The versions can and do differ substantially in the case of high $R$, where intrinsic decorrelation dominates and is modeled differently by the three versions.

Considering both low and high $R$, one might conclude that the RBD version provides the best overall agreement with numerical results, with very good agreement from low $R$ to $R=10$. (RBD is also the version that is easiest to use.) It is interesting to consider why RBD works well. As described in the Appendix, the RBD version uses an input displacement distribution based on low- $z$ (random ballistic) trajectories, the DD version is based on high- $z$ (diffusive) trajectories, and the ODE version uses a self-consistent displacement variance. While the ODE model would appear to be most reasonable, the numerical results for $R=10$ shed light on why RBD works best. DD makes substantial errors at low $z$ because of its use of high- $z$ diffusive displacements. While both RBD and ODE agree with the numerical result at low $z$, Corrsin's hypothesis involves some errors at high $R$ (Weinstock 1976; Vlad et al. 1998). These errors may be compounded by the self-consistent ODE model, for which the calculated displacement variance feeds back into the calculation. Thus, we find an increasing error in $D$ with increasing $z$ for the ODE version and better results for RBD. Even at the extreme value of $R=100$, RBD matches the numerical results to within $40 \%$.

At $R=100$, there is an interesting phenomenon of transient subdiffusion in the numerical results (see Figure 3). Strictly speaking, subdiffusion (superdiffusion) in the sense of a decreasing (increasing) $D(z)$ also occurs for $R=0.1$ and 1 , for which $D(z)$ oscillates because the Eulerian correlation function has negative values in certain $z$ ranges for our choice of a boxcar power spectrum as a function of $k_{z}$. The Corrsin-based theory can successfully model such oscillations. However, the transient subdiffusion in numerical results at $R=100$ occurred at lower values of $z$ where the Eulerian correlation was still positive, indicating that the Lagrangian correlation (which equals $d D / d z$ ) was negative, a feature that the Corrsin-based theory is unable to model. Previous work noted such transient subdiffusion when the FLRW was dominated by nearly 2D fluctuations, which mainly vary in the $x$ - and $y$-directions, and attributed such subdiffusion to trapping effects (Ruffolo et al. 2008; Ghilea et al. 2011). Similar behaviors have been reported from simulations of the mathematically analogous situation of trajectories of tracers in 2D hydrodynamic turbulence (Ottaviani 1992). We attribute both the transient subdiffusion and the disagreement with the Corrsin-based theory to trapping effects in quasi-2D fluctuations that are not addressed by the theory.

For a range of $R$ values, we determined the asymptotic diffusion coefficient $D_{\infty}$ by averaging over several parallel correlation lengths for a $z$-range such that $D(z)$ has only minor oscillations about an asymptotic level. In all cases, this did not extend beyond $z=L_{z} / 2$. Results for $D_{\infty}$ as a function of $R$ 


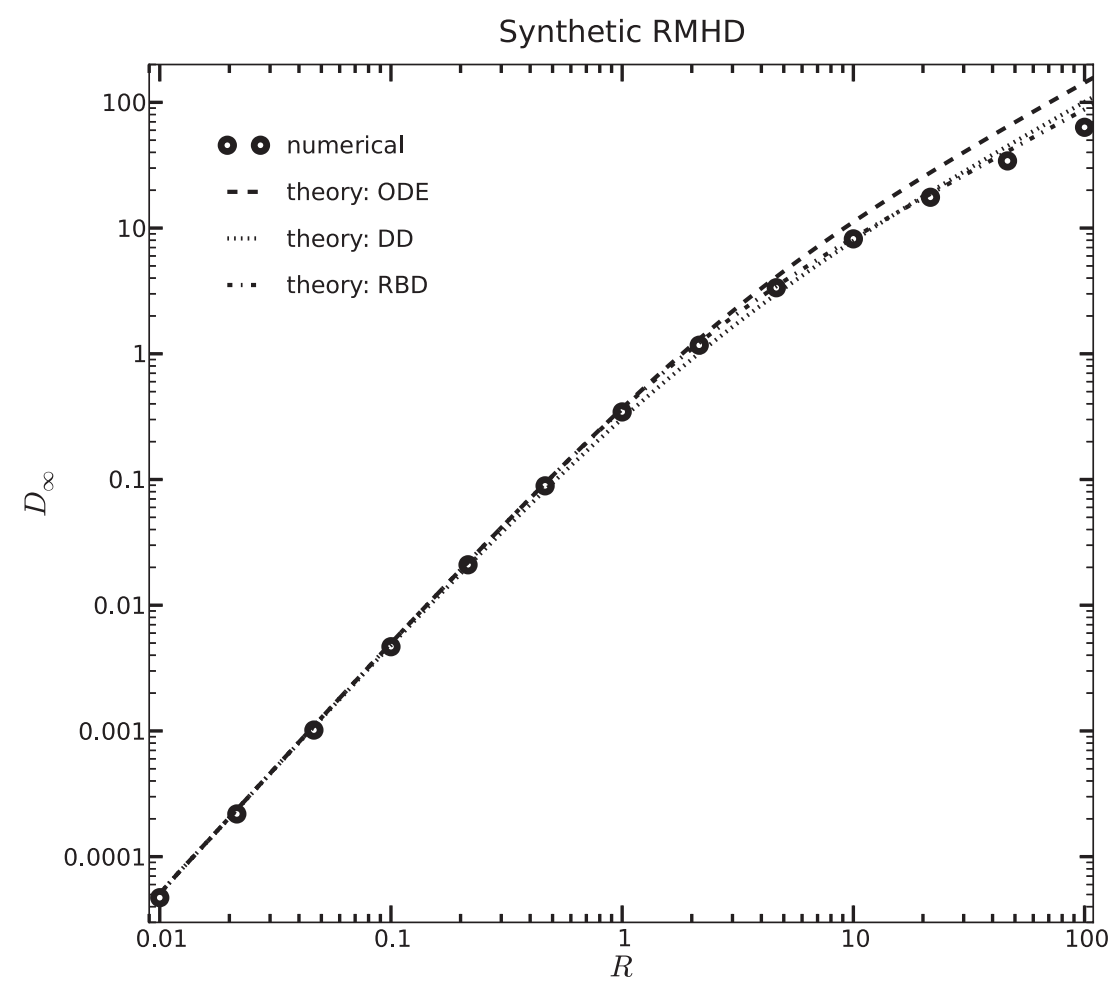

Figure 4. Asymptotic field line diffusion coefficient $D_{\infty}$ as a function of the Kubo number $R$ for synthetic RMHD turbulence, from numerical field line tracing (circles) and from theory (dashed lines). All versions of the theory provide an excellent match to numerical results in the quasi-linear regime $(R<1)$ and the RBD version also provides a very good match for $0.1 \lesssim R \lesssim 10$. There is some deviation for very high Kubo numbers, presumably due to trapping effects.

are shown in Figure 4, confirming the accuracy of the theory, and particularly the RBD version, up to $R=10$, with gradual divergence thereafter.

The scaling of $D$ with the Kubo number $R$ is of interest because different theories can have different predictions for the logarithmic derivative,

$$
\gamma \equiv \frac{R}{D_{\infty}} \frac{d D_{\infty}}{d R},
$$

which is a local power-law index such that $D_{\infty} \propto R^{\gamma}$. Figure 5 shows $\gamma$ as a function of $R$ for numerical results and the theory. ${ }^{7}$ These Corrsin-based theory results have $\gamma \approx 2$ at low $R$, indicating quasi-linear scaling, and $\gamma \approx 1$ at high $R$, which is called Bohm scaling. Our numerical results corroborate the theory well up to $R=10$, especially for the RBD version of the theory. For $R>10$, the numerical value of $\gamma$ decreases below 1 , which we attribute to trapping and/or percolation effects in quasi-2D fluctuations. Note that the percolation model predicts $\gamma=0.7$ (Isichenko 1991), and our numerical result is close to $\gamma=0.7$ at $R=100$. Our numerical result for $\gamma$ decreases more gradually as a function of $R$ than that of Hauff et al. (2010) (for isotropic turbulence that was stretched in $\mathbf{k}$-space), who found $\gamma \approx 1.2$ at $R=1$ and $0.7<\gamma<0.8$ for $10<R \leqslant 500$.

\subsection{RMHD Simulation}

We have also performed field line tracing for the dynamically evolved magnetic field obtained from RMHD simulation, for a snapshot of the magnetic field at $t=0.6$. The simulation introduces phase correlations that lead to physically realistic

\footnotetext{
7 In Figure 2 of Ruffolo \& Matthaeus (2013), the curve of $\gamma$ as a function of $R$ for ODE was plotted incorrectly; this is corrected in Figure 5 of the present work.
}

coherent structures and intermittency that were not present in the initial condition (the random-phase synthetic RMHD field). Our numerical results are compared with the theory results in Figure 6.

The Corrsin-based theory only considers the power spectrum of fluctuations and does not include phase correlations. To help distinguish the effects of phase correlations and other approximations made by the theory, we have also performed field line tracing on the RMHD simulation data with the phase correlations removed. We accomplish this phase randomization by fast Fourier transforming the RMHD data, then randomizing the complex phases while maintaining the same modal spectrum and the Hermitian reality condition, and performing an inverse fast Fourier transform. In this way, we have in hand two RMHD magnetic field data sets with the same power spectrum, and therefore the theory makes the same predictions for both data sets. Numerical results for the phase-randomized RMHD simulation data are also shown in Figure 6.

For $R=0.1$, the diffusion coefficient requires an extended distance $z$ (plotted in units of the correlation scale for this spectrum) to approach its asymptotic value. Such behavior could be called transient superdiffusion. In any case, this behavior is associated with the Eulerian correlation function and power spectrum of this RMHD snapshot field, and is matched very well by the Corrsin-based theory. There is a small but clear difference between the numerical results without and with phase randomization in Figure 6. As a check, for $R=0.1$ we numerically traced field lines for a second independent phase randomization and obtained essentially identical results for the two randomizations. The theory, which does not account for coherent structures, provides a particularly close match to numerical results for the phase-randomized fields. All versions of the theory calculate $D$ to well within $2 \%$ of those numerical results. DD is in extremely good agreement with the random-phase 


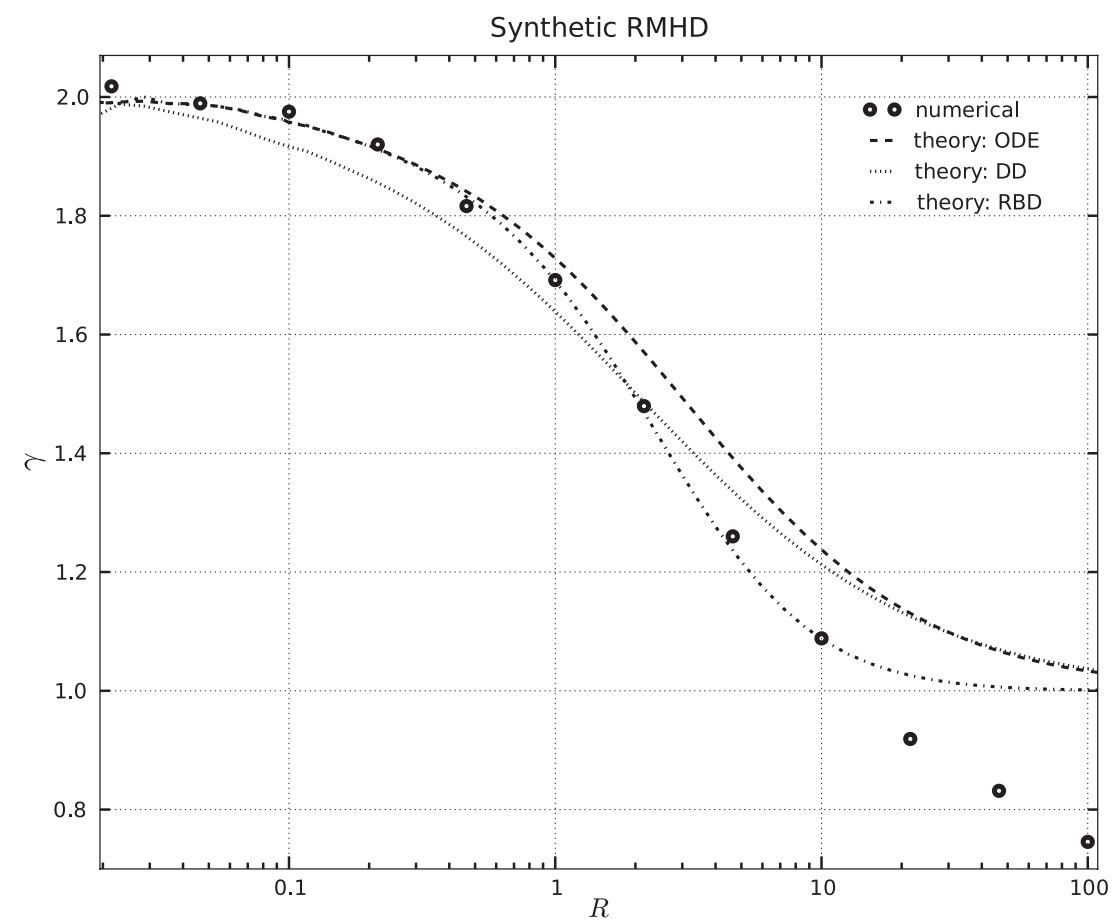

Figure 5. Local power-law index $\gamma \equiv\left(R / D_{\infty}\right)\left(d D_{\infty} / d R\right)$ of $D_{\infty}$ vs. the Kubo number $R$ for synthetic RMHD turbulence. For each version of the theory, the index tends from 2 (for quasi-linear diffusion) at low $R$ toward 1 (for Bohm diffusion) at high $R$. The numerical results corroborate the RBD theory up to $R=10$, and decline beyond that, presumably due to trapping effects.
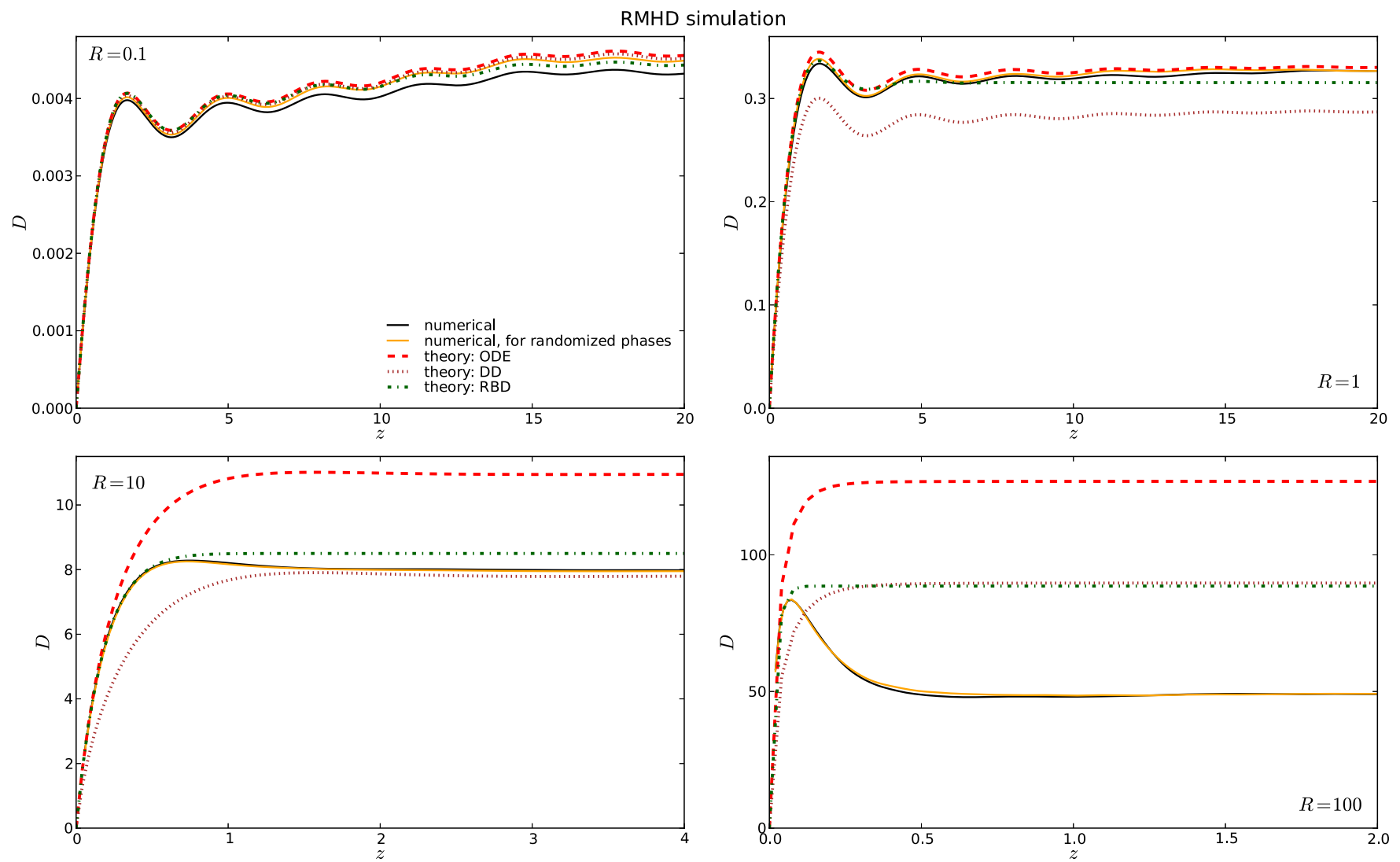

Figure 6. Running field line diffusion coefficient $D$ as a function of $z$ for a simulation of RMHD turbulence at various Kubo numbers $R$, from numerical field line tracing (solid lines) and from theory (dashed lines) using the simulated power spectrum corresponding to Figure 2. Note the different $z$ scales for different panels. Numerical results are for the simulated RMHD field (dark solid line) and that field after randomizing the Fourier phases (light solid line), which removes the coherent structures and intermittency of RMHD but preserves the power spectrum. For $R=0.1$, retaining the coherent structures gives a slightly lower field line diffusion coefficient. The theory is in excellent agreement with the numerical results, particularly for the phase-randomized field because the theory does not account for phase coherence. For $R=1$ and 10, the coherent structures have very little effect on the numerical results, and the RBD version of the theory is in very good agreement with those. For $R=100$ the theory has poorer agreement with numerical results, though the RBD and DD versions remain within a factor of two of the numerical RMHD simulation result. 
simulation data, within $0.5 \%$ over six or more parallel correlation lengths. Thus, the Corrsin-based theory can model the FLRW for a low Kubo number, even for a dynamically derived field with a power spectrum that does not have a simple analytic form. We also see that the coherent structures in a realistic simulation of magnetic turbulence can affect the FLRW.

In Figure 6, we see that for $R=1,10$, and 100, there is no significant difference between the two solid lines, i.e., no noticeable effect of the phase coherence. Overall, for low to moderate values of $R$ the non-linear diffusion models agree with the numerical field line tracing, especially for the RBD version, which again provides a good match to the numerical results for $R \leqslant 10$. We find transient subdiffusion for $R \geqslant 10$, due to trapping effects.

At $R=100$, the theory has poorer agreement with numerical results, which we again attribute to trapping effects that are not treated by the theory, although the RBD and DD versions remain within a factor of two of the numerical RMHD simulation result.

\section{DISCUSSION AND CONCLUSIONS}

Based on statistics accumulated from collections of numerically determined magnetic field lines in transverse anisotropic models of turbulence, we have been able to verify that a recently developed theory (Ruffolo \& Matthaeus 2013) of the FLRW for such fields is accurate over a wide range of Kubo numbers. The model magnetic fields that we have employed are both a synthetic field that can be used as initial data for a RMHD simulation, and also output data from RMHD simulation. The latter differ in that the magnetic field has been able to develop characteristic coherent structures through non-linear dynamical evolution, resulting in scale-dependent anisotropy (Oughton et al. 1994) that is qualitatively consistent with models of critical balance (Goldreich \& Sridhar 1995). Such scaledependent anisotropy has little effect on the FLRW, which is mainly sensitive to the power spectrum at low $k$, corresponding to scales larger than the outer scale of the turbulence (Ruffolo \& Matthaeus 2013).

In addition to our study of the FLRW for synthetic initial data and a snapshot from a RMHD simulation, the same analysis was also performed on a phase-randomized version of the snapshot field, for which the spectrum was kept fixed. By construction, both the initial data and the phase-randomized final state lack coherent structures and exhibit Gaussian statistics. The comparison with the non-phase-randomized final state has shown that the presence of coherent structures does not have a large impact on the statistics of field line transport, at least at the level of the statistics related to determination of the diffusion coefficient.

In comparing the ensemble of field line trajectories with the diffusion theory, we examined three alternative formulations by Ruffolo \& Matthaeus (2013) in which the decorrelation responsible for saturation of the Taylor-Green-Kubo (TGK) integral takes place in different ways. It is useful to think of these alternatives as different closures of the diffusion problem. The simplest case is quasi-linear theory, which neglects transverse displacements altogether, and is well known to work well at low Kubo number (Kadomtsev \& Pogutse 1979). Here we examine three closures that allow transverse displacements, involving different assumptions about how the Lagrangian decorrelation behaves (Snodin et al. 2013). All of these use Corrsin's independence hypothesis and assume a Gaussian displacement distribution. In DD, the variances are considered diffusive at all positions along the trajectory. RBD uses a variance based on directionally randomized ballistic (linear) trajectories. In the ODE self-closure, a consistent pair of ODEs determines the variance in the TGK integral. A major conclusion of the present study is that all three closures work reasonably well for Kubo numbers ranging from $R \ll 1$ to values well beyond $R=1$, and that RBD works best. In fact reasonable results are obtained up until $R=100$. We are led to the conclusion that the Corrsin hypothesis is valid beyond the small $R$ limit that has sometimes been assumed to be its region of validity.

The ability to calculate the magnetic FLRW for RMHD turbulence has implications for other astrophysical problems. Theories of energetic charged particle transport perpendicular to a mean field have included the field line diffusion coefficient since the groundbreaking work of Jokipii \& Parker (1968). More recently, the non-linear guiding center theory of Matthaeus et al. (2003) and derived theories (e.g., Ruffolo et al. 2012) are closely related to the calculation of the FLRW, and have successfully explained numerical simulation results for the perpendicular diffusion of energetic particles. Furthermore, heat transport is often mediated by the transport of electrons along magnetic field lines, and is critical for understanding the solar transition region and corona, which is a physical situation where RMHD provides a reasonable description of the turbulence. Kittinaradorn et al. (2009) showed that "moss" patterns of EUV emission in the solar transition region can be understood in terms of heat transport mediated by spatially dependent magnetic connectivity in hot coronal loops. Galloway et al. (2006) considered the importance of the separation of neighboring field lines, which is related to field line diffusion, for heat transport in coronal loops. Note, however, that the spatial connectivity of magnetic field lines is only part of the story, as Lazarian (2006) has calculated this effect on heat transport and finds that it is often dominated by dynamical effects of the turbulence.

We have established here that diffusive theories of FLRW are reasonably accurate for RMHD, which is a highly anisotropic quasi-2D model. We expect that the formulation of diffusion adopted here (Ruffolo \& Matthaeus 2013) should be readily adaptable to related models such as those using critical balance (Goldreich \& Sridhar 1995). There are also related problems that may be addressed using these methods and asymptotic diffusion theories. These include, for example, the related problems of field line separation (Jokipii 1973; Ruffolo et al. 2004) in RMHD and the time-dependent FLRW (e.g., Rappazzo et al. 2012). We are currently pursuing those theoretical applications and intend to report on them in the near future. Finally, here we have largely examined the asymptotic character of the field line transport. The transition to diffusion is also a problem of substantial interest, in which one may examine, in the context of RMHD, potential regimes of free-streaming, Richardson diffusion, and the exponential instability phase of transport. We have not emphasized these in the present case, but anticipate more detailed treatments in the future.

This work was supported by a postdoctoral fellowship from Mahidol University, the Thailand Research Fund, POR Calabria FSE-2007/2013, the U.S. NSF (AGS-1063439 and SHINE AGS-1156094), NASA (Heliophysics Theory NNX08AI47G and NNX11AJ44G), by the Solar Probe Plus Project through the ISIS Theory team, and by EU Marie Curie Project FP7 PIRSES2010-269297 "Turboplasmas" at Università della Calabria. We acknowledge useful discussions with Vincenzo Carbone and Pierluigi Veltri at Università della Calabria. 


\section{APPENDIX}

\section{THEORETICAL EXPRESSIONS FOR THE FIELD LINE DIFFUSION COEFFICIENT}

Because the present work compares the results of field line tracing in model and simulated RMHD fields with theoretical expressions from Ruffolo \& Matthaeus (2013), those expressions are reproduced here. Consider the dimensionless variables $x^{\prime}=x / \ell_{\perp}$ and $z^{\prime}=K z$, where $K$ is the maximum $k_{z}$ excited in the synthetic field (initial condition) employed here (see Equation (7)). Derived quantities include the variance of the displacement distribution $V \equiv\left\langle\Delta x^{2}\right\rangle$ and the dimensionless variance $V^{\prime}=V / \ell_{\perp}^{2}$, as well as the running diffusion coefficient $D \equiv(1 / 2)(d V / d z)$ and the dimensionless quantity $D^{\prime}=D /\left(K \ell_{\perp}^{2}\right)$. After such scaling, for a given type of fluctuation field, $V^{\prime}$ and $D^{\prime}$ depend only on the Kubo number,

$$
R=\frac{b}{B_{0}} \frac{\ell_{c}}{\ell_{\perp}}=\frac{b}{B_{0}} \frac{\pi}{2 K \ell_{\perp}} .
$$

From Equation (9) of Snodin et al. (2013), we have

$$
\frac{d D}{d z}=\frac{1}{B_{0}^{2}}\left\langle b_{x}(0,0) b_{x}\left[\mathbf{x}_{\perp}(z), z\right]\right\rangle
$$

where $\mathbf{x}_{\perp}(z)$ is a Lagrangian displacement along the field line. We use Corrsin's hypothesis (Corrsin 1959) to relate this Lagrangian correlation to the Eulerian correlation function $R_{x x} \equiv\left\langle b_{x}(0,0) b_{x}\left(\mathbf{x}_{\perp}, z\right)\right\rangle$, averaged using the conditional probability $P\left(\mathbf{x}_{\perp} \mid z\right)$ of finding a displacement $\mathbf{x}_{\perp}$ after a distance $z$ :

$$
\left\langle b_{x}(0,0) b_{x}\left[\mathbf{x}_{\perp}(z), z\right]\right\rangle=\int R_{x x}\left(\mathbf{x}_{\perp}, z\right) P\left(\mathbf{x}_{\perp} \mid z\right) d \mathbf{x}_{\perp} .
$$

Expressing the correlation function $R_{x x}\left(\mathbf{x}_{\perp}, z\right)$ in terms of its Fourier transform, the power spectrum $P_{x x}(\mathbf{k})$, using the power spectrum for RMHD corresponding to Equation (7), and assuming a Gaussian displacement distribution, one obtains

$$
\begin{gathered}
\frac{d V^{\prime}}{d z^{\prime}}=2 D^{\prime} \\
\frac{d D^{\prime}}{d z^{\prime}}=\frac{2}{\pi^{2}} R^{2} \frac{\int k_{\perp}^{\prime 2} A\left(k_{\perp}^{\prime}\right) e^{-\frac{1}{2} k_{\perp}^{\prime 2} \sigma^{\prime 2}\left(z^{\prime}\right)} d \mathbf{k}_{\perp}^{\prime}}{\int k_{\perp}^{\prime 2} A\left(k_{\perp}^{\prime}\right) d \mathbf{k}_{\perp}^{\prime}} \frac{\sin z^{\prime}}{z^{\prime}},
\end{gathered}
$$

with $V^{\prime}(0)=D^{\prime}(0)=0$, where $A\left(k_{\perp}^{\prime}\right)$ is the power spectrum of the magnetic potential function $a$ (see Sections 2.1 and 3.1), and $\sigma^{\prime}$ represents the dimensionless width of the perpendicular displacement distribution after a parallel displacement $z^{\prime}$, so that $\sigma^{\prime 2}$ is an estimate of $V(z)$. Note that $k_{\perp}^{\prime 2} A\left(k_{\perp}^{\prime}\right)$ is proportional to the total (trace) power spectrum of $\mathbf{b}$.

Three useful closures of this system of differential equations, involving different estimates $\sigma^{\prime 2}$, lead to three versions of the theory. Ordered from simple to complex expressions, these are as follows.

1. Random ballistic decorrelation (RBD; Ghilea et al. 2011): Use $\sigma^{2}=(1 / 2)\left(b^{2} / B_{0}^{2}\right) z^{2}$ or $\sigma^{\prime 2}=\left(2 / \pi^{2}\right) R^{2} z^{\prime 2}$, based on the low $z$ behavior of the displacement distribution (see Figure 1). Equation (A5) can be directly integrated to obtain $D^{\prime}\left(z^{\prime}\right)$. An analytic expression for asymptotic diffusion is

$$
D_{\infty}^{\prime}=\frac{R^{2}}{\pi} \frac{\int k_{\perp}^{\prime 2} A\left(k_{\perp}^{\prime}\right) \operatorname{erf}\left(\pi / 2 R k_{\perp}^{\prime}\right) d \mathbf{k}_{\perp}^{\prime}}{\int k_{\perp}^{\prime 2} A\left(k_{\perp}^{\prime}\right) d \mathbf{k}_{\perp}^{\prime}} .
$$

The limiting expressions for $R \ll 1$ are $D_{\infty}=$ $(1 / 2)\left(b / B_{0}\right)^{2} \ell_{c}$ and $D_{\infty}^{\prime}=R^{2} / \pi$ (quasi-linear diffusion; Jokipii \& Parker 1969a) and those for $R \gg 1$ are $D_{\infty}=$ $(\sqrt{\pi} / 2)\left(b / B_{0}\right) \ell_{\perp}$ and $D_{\infty}^{\prime}=R / \sqrt{\pi}$.

2. Diffusive decorrelation (DD; Salu \& Montgomery 1977; Matthaeus et al. 1995): Use $\sigma^{\prime 2}=2 D_{\infty}^{\prime} z^{\prime}$, based on the high- $z$ behavior of the displacement distribution (see Figure 1). Then we can integrate Equation (A5) for $z^{\prime}$ from 0 to $\infty$ to obtain

$$
D_{\infty}^{\prime}=\frac{2}{\pi^{2}} R^{2} \frac{\int k_{\perp}^{\prime 2} A\left(k_{\perp}^{\prime}\right) \tan ^{-1}\left(1 / k_{\perp}^{\prime 2} D_{\infty}^{\prime}\right) d \mathbf{k}_{\perp}^{\prime}}{\int k_{\perp}^{\prime 2} A\left(k_{\perp}^{\prime}\right) d \mathbf{k}_{\perp}^{\prime}},
$$

which represents an implicit equation for $D_{\infty}^{\prime}$. After solving that to determine $D_{\infty}^{\prime}$, Equation (A5) can be integrated to any desired $z^{\prime}$. The limiting expression for $R \ll 1$ is again $D_{\infty}^{\prime}=R^{2} / \pi$ (quasi-linear diffusion) and for $R \gg 1$ we now have $D_{\infty}=\left(b / B_{0}\right)(\tilde{\lambda} / \sqrt{2})$ and $D_{\infty}^{\prime}=\left(\sqrt{2} \tilde{\lambda} / \pi \ell_{\perp}\right) R$ (Bohm diffusion), where $\tilde{\lambda}$ is the ultrascale (Matthaeus et al. 1995), following the definition of Ruffolo et al. (2004).

3. Ordinary differential equation (ODE; Snodin et al. 2013, and references therein): Identify $\sigma^{\prime 2}$ with the variance $V^{\prime}$, which is self-consistent but in practice can amplify approximation errors at low $z^{\prime}$. Now Equations (A4) and (A5) are coupled to form a second-order ODE, which can readily be solved numerically. The limiting expression for $R \ll 1$ is once again $D_{\infty}^{\prime}=R^{2} / \pi$ (quasi-linear diffusion) and for $R \gg 1$ we now have $D_{\infty}=\left(b / B_{0}\right) \tilde{\lambda}$ and $D_{\infty}^{\prime}=\left(2 \tilde{\lambda} / \pi \ell_{\perp}\right) R$ (Bohm diffusion), which is a factor of $\sqrt{2}$ higher than the DD result.

Ruffolo \& Matthaeus (2013) proposed using RBD because its explicit expressions are the simplest to use. Furthermore, in the present work, we find that RBD is typically closest to the simulation results.

The above formulae are for a model RMHD power spectrum of the form of Equation (7). In the present work, we also want to apply this theoretical framework for the RMHD simulation spectrum, which does not exactly match that model. We therefore use an expression for a more general power spectrum of transverse fluctuations (which was Equation (16) of Snodin et al. (2013) and Equation (49) of Ruffolo \& Matthaeus 2013), replacing the integrals with sums over the discrete Fourier grid for which the spectrum is determined. For consistency, we use the same procedure for comparison with numerical results from the synthetic RMHD model and the RMHD simulation. For the synthetic model, the discrete sum over $k_{z}$ gives results with very little difference from continuous integration, so in that sense the theory results shown in Figures 3-5 do represent the results of Equations (A4) to (A7).

Note further that all of the above limiting expressions for low or high $R$ are more general and apply to any transverse fluctuation field with finite correlation scales. To see this, consider the Lagrangian correlation function in Equation (A3). In the quasi-1D limit $R \ll 1$, this decorrelates due to the $z$-dependence of $R_{x x}$ while $\left|\mathbf{x}_{\perp}\right| \ll \ell_{\perp}$, and $P\left(\mathbf{x}_{\perp} \mid z\right)$ can be approximated as $\delta\left(\mathbf{x}_{\perp}\right)$, yielding the quasi-linear approximation. In the quasi-2D limit $R \gg 1$, it decorrelates due to $(x, y)$ excursions while $z \ll \ell_{c}$, so that $R_{x x}\left(\mathbf{x}_{\perp}, z\right)$ can be replaced by $R_{x x}\left(\mathbf{x}_{\perp}, 0\right)$, yielding a limiting expression for each version of the theory as presented above. 


\section{REFERENCES}

Cash, J. R., \& Karp, A. H. 1990, ACM Trans. Math. Softw., 16, 201 Chandran, B. D. G., \& Cowley, S. C. 1998, PhRvL, 80, 3077 Cho, J., \& Lazarian, A. 2002, PhRvL, 88, 245001

Cho, J., Lazarian, A., \& Vishniac, E. T. 2002, ApJ, 564, 291

Corrsin, S. 1959, in Atmospheric Diffusion and Air Pollution, ed. F. Frenkel \&

P. Sheppard (Advances in Geophysics, Vol. 6; New York: Academic), 161

Dalena, S., Chuychai, P., Mace, R. L., et al. 2012, CoPhC, 183, 1974

Dmitruk, P., \& Gómez, D. O. 1997, ApJL, 484, L83

Dmitruk, P., \& Matthaeus, W. H. 2003, ApJ, 597, 1097

Dmitruk, P., Matthaeus, W. H., \& Oughton, S. 2005, PhP1, 12, 112304

Dobrowolny, M., Mangeney, A., \& Veltri, P. 1980, A\&A, 83, 26

Einaudi, G., Velli, M., Politano, H., \& Pouquet, A. 1996, ApJL, 457, L113

Galloway, R. K., Helander, P., \& MacKinnon, A. L. 2006, ApJ, 646, 615

Ghilea, M. C., Ruffolo, D., Chuychai, P., et al. 2011, ApJ, 741, 16

Giacalone, J., \& Jokipii, J. R. 1996, JGR, 101, 11095

Giacalone, J., \& Jokipii, J. R. 1999, ApJ, 520, 204

Giacalone, J., Jokipii, J. R., \& Mazur, J. E. 2000, ApJL, 532, L75

Goldreich, P., \& Sridhar, S. 1995, ApJ, 438, 763

Hauff, T., Jenko, F., Shalchi, A., \& Schlickeiser, R. 2010, ApJ, 711, 997

Isichenko, M. B. 1991, PPCF, 33, 809

Jokipii, J. R. 1966, ApJ, 146, 480

Jokipii, J. R. 1973, ApJ, 183, 1029

Jokipii, J. R., \& Parker, E. N. 1968, PhRvL, 21, 44

Jokipii, J. R., \& Parker, E. N. 1969a, ApJ, 155, 777

Jokipii, J. R., \& Parker, E. N. 1969b, ApJ, 155, 799

Kadomtsev, B. B., \& Pogutse, O. P. 1979, in Plasma Physics and Controlled Nuclear Fusion Research 1978: Proc. Seventh Internat. Conf. (Vienna: IAEA), 649

Kittinaradorn, R., Ruffolo, D., \& Matthaeus, W. H. 2009, ApJL, 702, L138

Kowal, G., \& Lazarian, A. 2010, ApJ, 720, 742

Lazarian, A. 2006, ApJL, 645, L25

Lazarian, A., \& Vishniac, E. T. 1999, ApJ, 517, 700

Lithwick, Y., \& Goldreich, P. 2001, ApJ, 562, 279
Matthaeus, W. H., Bieber, J. W., Ruffolo, D., Chuychai, P., \& Minnie, J. 2007, ApJ, 667, 956

Matthaeus, W. H., Gray, P. C., Pontius, D. H., Jr., \& Bieber, J. W. 1995, PhRvL, 75,2136

Matthaeus, W. H., Qin, G., Bieber, J. W., \& Zank, G. 2003, ApJL, 590, L53

Montgomery, D. 1982, PhST, 1, 83

Negrea, M., Petrisor, I., \& Weyssow, B. 2007, PPCF, 49, 1767

Neuer, M., \& Spatschek, K. H. 2006, PhRvE, 74, 036401

Ottaviani, M. 1992, EPL, 20, 111

Oughton, S., Dmitruk, P., \& Matthaeus, W. H. 2004, PhP1, 11, 2214

Oughton, S., Priest, E. R., \& Matthaeus, W. H. 1994, JFM, 280, 95

Rappazzo, A. F., Matthaeus, W. H., Ruffolo, D., Servidio, S., \& Velli, M. 2012, ApJL, 758, L14

Rechester, A. B., \& Rosenbluth, M. M. 1978, PhRvL, 40, 38

Reuss, J.-D., \& Misguich, J. H. 1996, PhRvE, 54, 1857

Ruffolo, D., Chuychai, P., \& Matthaeus, W. H. 2006, ApJ, 644, 971

Ruffolo, D., Chuychai, P., Wongpan, P., et al. 2008, ApJ, 686, 1231

Ruffolo, D., \& Matthaeus, W. H. 2013, PhPl, 20, 012308

Ruffolo, D., Matthaeus, W. H., \& Chuychai, P. 2003, ApJL, 597, L169

Ruffolo, D., Matthaeus, W. H., \& Chuychai, P. 2004, ApJ, 614, 420

Ruffolo, D., Pianpanit, T., Matthaeus, W. H., \& Chuychai, P. 2012, ApJL, 747, L34

Salu, Y., \& Montgomery, D. C. 1977, PhFl, 20, 1

Shalchi, A., \& Kolly, A. 2013, MNRAS, 431, 1923

Snodin, A. P., Ruffolo, D., \& Matthaeus, W. H. 2013, ApJ, 762, 66

Strauss, H. R. 1976, PhFl, 19, 134

Taylor, J. B., \& McNamara, B. 1971, PhFl, 14, 1492

Veltri, P. 1999, PPCF, 41, A787

Vlad, M., Spineanu, F., Misguich, J. H., \& Balescu, R. 1998, PhRvE, 58, 7359

Weinhorst, B., Shalchi, A., \& Fichtner, H. 2008, ApJ, 677, 671

Weinstock, J. 1976, PhFl, 19, 1702

Zank, G. P., \& Matthaeus, W. H. 1992, JPIPh, 48, 85

Zimbardo, G., Pommois, P., \& Veltri, P. 2004, JGR, 109, A02113

Zimbardo, G., \& Veltri, P. 1995, PhRvE, 51, 1412

Zimbardo, G., Veltri, P., \& Pommois, P. 2000, PhRvE, 61, 1940 